\title{
Exact multi-twist solutions to the Belavin-Polyakov equation and applications to magnetic systems*
}

\author{
Raphael Blumenfeld $\dagger \S$ and Radha Balakrishnan $\ddagger$ \\ $\uparrow$ Theoretical Division and CNLS, Los Alamos National Laboratory, Los Alamos, NM 87545, \\ USA \\ \$ The Institute of Mathematical Sciences, CIT Campus, Madras 600 113, India
}

Received 24 August 1999

Abstract. The Belavin-Polyakov equation $t_{u}=t \times t_{s}$ with $t^{2}=1$ has been shown recently to describe the evolution of a wide class of space curves, and has several physical applications. Here, we obtain a hierarchy of exact multi-twist solutions for this nonlinear system. As an illustration, we apply our results to continuum magnetic models. When $u$ and $s$ denote temporal and spatial variables respectively, these twists describe very low-energy domain walls travelling along an antiferromagnetic spin chain. When they denote independent spatial variables, the solutions represent twists in the static configuration (texture) of a two-dimensional ferromagnet.

\section{Introduction}

The study of space-curve evolution can be used in many physical applications such as the motion of a vortex filament, kinematics of a polymer chain, interface dynamics, low-dimensional magnets etc. In general, curve evolution can be described [1] by the following two sets of first-order ODEs for the unit orthogonal triad of vectors $(t, n, b)$ :

$$
\boldsymbol{t}_{s}=K \boldsymbol{n} \quad \boldsymbol{n}_{s}=-K \boldsymbol{t}+\tau \boldsymbol{b} \quad \boldsymbol{b}_{s}=-\tau \boldsymbol{n}
$$

and

$$
\boldsymbol{t}_{u}=g \boldsymbol{n}+h \boldsymbol{b} \quad \boldsymbol{n}_{u}=-g \boldsymbol{t}+\tau_{0} \boldsymbol{b} \quad \boldsymbol{b}_{u}=-h \boldsymbol{t}-\tau_{0} \boldsymbol{n} .
$$

Here, $\boldsymbol{t}, \boldsymbol{n}$ and $\boldsymbol{b}$ denote, respectively, the tangent to the curve, the normal and binormal vectors. The arc length, $s$, and another variable, $u$ (say, time) are independent. The subscripts $s$ and $u$ stand for the respective partial derivatives. Equations (1) are the usual Frenet-Serret [1] equations, with spatial curvature $K=\left(t_{s} \cdot t_{s}\right)^{1 / 2}$ and torsion $\tau=t \cdot\left(t_{s} \times t_{s s}\right) / K^{2}$. Likewise, the temporal curvatures $g$ and $h$ and the temporal torsion $\tau_{0}$ can be appropriately defined by using equations (2). We have recently shown [2] that if $g=0$ while $K$ and $h$ are non-zero, the curve evolution reduces to the form

$$
\boldsymbol{t}_{s}=(K / h)\left(\boldsymbol{t}_{u} \times \boldsymbol{t}\right) .
$$

We named this the modified Belavin-Polyakov equation (MBPE), owing to the fact that if $K / h$ is a separable function of $u$ and $s$, say $[G(u) / F(s)]$, then one can transform to a new

* We dedicate this paper to the memory of Sergio de Menezes who pointed out possible instabilities of the single-twist dynamical solution in the context of the discrete AFM chain. Sergio's untimely demise cut short many of his ideas regarding extensions of this work. R Blumenfeld gratefully acknowledges funding by the Director's Fellowship at Los Alamos National Laboratory.

$\S$ Present address: Cavendish Laboratory, Cambridge University, Madingley Road, Cambridge CB3 0HE, UK. 
set of variables, $u^{\prime}=\int^{u} G(u) \mathrm{d} u$ and $s^{\prime}=\int^{s} F(s) \mathrm{d} s$, to obtain the usual Belavin-Polyakov equation (BPE) [3]

$$
t_{u^{\prime}}=t \times t_{s^{\prime}}
$$

When $h=K$, equation (3) is exactly the BPE in terms of the actual space-time variables. While the derivation of (3) for general curves may be found in [2] it is instructive to recall how it is obtained for $g=0$. From equations (1) and (2) we have, quite generally,

$$
\boldsymbol{t}_{s} \cdot \boldsymbol{t}_{u}=K g \quad \boldsymbol{t}_{s} \cdot \boldsymbol{t}_{s}=K^{2} \quad \boldsymbol{t}_{u} \cdot \boldsymbol{t}_{u}=g^{2}+h^{2} .
$$

and

$$
t_{s} \times t_{u}=K h t
$$

Taking the cross product of the latter relation from the left with $\boldsymbol{t}_{s}$, and using the former, we find

$$
g t_{s}-K t_{u}=h\left(t_{s} \times t\right) .
$$

When $g=0$, (7) reduces to equation (6).

The BPE first appeared in the context of the nonlinear sigma model field theory and in the static two-dimensional classical ferromagnet, where both $s$ and $u$ are spatial coordinates. By using complex variables, it was shown to support multi-instanton [3] solutions. More recently, it has been found to describe the very low-energy dynamics of a classical one-dimensional antiferromagnet, in which context $s$ is a spatial coordinate that runs along the chain and $u$ is time. Searching for travelling wave solutions for $t$, it was possible to obtain a single-twist solution [4]. This method, however, could not be generalized to yield multi-twists. In this paper, we find a new transformation that allows us to identify a novel hierarchy of travelling multi-twist solutions to this equation.

The BPE turns out to be particularly useful in describing low-dimensional magnetic systems. Here, we discuss this application in some detail and interpret the physical significance of the multi-twists in these systems. The paper is organized as follows. In section 2 we outline how the BPE arises in magnetic systems and introduce a transformation that converts this vectorial equation into Cauchy-Riemann relations (CRRs) for two scalar variables. In section 3 we use complex variables to obtain, in addition to the well known multi-instantons [3], a class of non-travelling twists. In section 4 we use moving coordinates (real variables) first to rederive the already known travelling single twist [4] and then to obtain, for the first time, a bi-twist solution in this system. This solution describes a twist and an anti-twist moving apart. The twists are related to harmonic functions, which gives insight into their nature and behaviour. In section 5 we generalize these results to show how a hierarchy of multi-twists can be obtained using a systematic analysis. The hierarchy is labelled by an integer $N$ that can be interpreted physically as the maximal number of twists in the system. We discuss a particular subset of these solutions in the asymptotic regime and identify the first two members of the hierarchy as the single twist and bi-twist of section 4 . In continuum magnetic systems, the $N$-twists represent $N$ moving domain walls along the antiferromagnetic (AFM) chain, with each wall possessing a spin-wave like mode within the wall thickness. This new class of solutions is markedly distinct (both physically and mathematically) from the known multi-instantons [3]. Further, these correspond to static spin configurations (textures) in two-dimensional ferromagnets. The paper concludes with a summary and discussion in section 6 . 


\section{Applications to magnetic systems}

We begin by explaining briefly how the BPE becomes applicable in the continuum description of magnetic chains modelled by the classical isotropic Heisenberg exchange Hamiltonian,

$$
H=-J \sum_{n} S_{n} \cdot S_{n+1} \text {. }
$$

Here, $J$ is a constant nearest-neighbour interaction, $\boldsymbol{S}_{n}$ is the spin at the $n$th site and $\left(\boldsymbol{S}_{n}\right)^{2} \equiv S^{2}=$ constant. The spin evolution equation can be found [5] from

$$
\dot{S}_{n}=\left\{H, S_{n}\right\}
$$

where the curly brackets denote an appropriately defined Poisson bracket for the spin system. This leads to

$$
\dot{S}_{n}=J S_{n} \times\left(S_{n+1}+S_{n-1}\right)
$$

Equation (8) holds regardless of the sign of $J$. At this stage, we must distinguish between the ferromagnetic (FM) and AFM cases. In the former $(J>0)$ the low-energy spin orientations vary slowly over distances of the order of the lattice separation $a$, and the continuum version of equation (8) is obtained directly by using a Taylor expansion, $\boldsymbol{S}_{n \pm 1} \rightarrow$ $\boldsymbol{S}(x, t) \pm a \boldsymbol{S}_{x}+\left(a^{2} / 2\right) \boldsymbol{S}_{x x}+\cdots$, which yields the well known Landau-Lifshitz equation

$$
\boldsymbol{S}_{t}=\boldsymbol{S} \times \boldsymbol{S}_{x x}
$$

where $x \rightarrow x / a, t \rightarrow J S t$ are redefined in dimensionless form and $S^{2}=1$. The subscripts $x$ and $t$ represent partial derivatives. Equation (9) supports pulse-type solitary waves [5] and has been studied extensively. In particular, it is known to be integrable and to have $N$-soliton solutions [6].

In contrast, the study of the dynamics of isotropic AFM chains $(J<0)$ is less complete (see, for example, the review [7]) and the continuum low-energy analysis is complicated by the need to separate the even, $\left\{\boldsymbol{S}_{e}\right\}$, and the odd, $\left\{\boldsymbol{S}_{o}\right\}$, sublattices. Let us now define new orthogonal unit vectors $\boldsymbol{\eta}$ (the staggered magnetization) and $\boldsymbol{\xi}$ (the effective magnetization) via

$$
\boldsymbol{S}_{o}-\boldsymbol{S}_{e}=2 S \sqrt{1-\epsilon^{2}} \boldsymbol{\eta}(x, t) \quad \boldsymbol{S}_{o}+\boldsymbol{S}_{e}=2 S \epsilon \boldsymbol{\xi}(x, t)
$$

where $2 \epsilon^{2}=1+\left(\boldsymbol{S}_{o} \cdot \boldsymbol{S}_{e}\right) / S^{2}$, which are coupled through equation (8). At low energy $\epsilon \ll 1$ and, to zero order in $\epsilon$, the equations decouple to yield [4]

$$
\boldsymbol{\eta}_{t}=\left(\boldsymbol{\eta}_{x} \times \boldsymbol{\eta}\right)
$$

where $x \rightarrow x / 2 a$ and $t \rightarrow J S t$. Thus, equation (11) is just the BPE (equation (4)), when $\eta$ is identified with $t$.

Before analysing equation (11) we point out a connection with the two-dimensional static ferromagnetic case. The equation of motion of the latter is

$$
\boldsymbol{S}_{t}=\boldsymbol{S} \times \nabla^{2} \boldsymbol{S}=0
$$

which is obtained from the two-dimensional analogue of equation (9). It can be readily shown that this equation is solved by all the solutions of the first-order equation

$$
\boldsymbol{S}_{y}=\boldsymbol{S}_{x} \times \boldsymbol{S} .
$$

The latter equation is identical to the BPE when $\boldsymbol{\eta} \rightarrow \boldsymbol{S}$ and $t \rightarrow y$. The connection between the two systems is useful for obtaining static solutions for the planar FM from the dynamical solutions for the AFM and vice versa (although care should be taken in transforming initial conditions to boundary conditions). In addition, when suitably interpreted, such solutions would also apply to the evolution of curves as described by equation (4). 
To make progress, write $\boldsymbol{\eta}$ (the tangent or effective spin vector) in terms of its polar angle $\theta$ and azimuthal angle $\phi$ :

$$
\boldsymbol{\eta}=(\sin \theta \cos \phi, \sin \theta \sin \phi, \cos \theta) .
$$

Substituting this into equation (11), we obtain

$$
\theta_{x}=-\sin \theta \phi_{t} \quad \theta_{t}=\sin \theta \phi_{x} .
$$

We now transform the angle variable $\theta$ to $\psi$ by using

$$
\psi \equiv \ln \sqrt{(1+\cos \theta) /(1-\cos \theta)} \equiv \ln \cot (\theta / 2) \text {. }
$$

This reduces relations (13) to

$$
\psi_{x}=\phi_{t} \quad \psi_{t}=-\phi_{x}
$$

with $\psi_{x x}+\psi_{t t}=\phi_{x x}+\phi_{t t}=0$. In terms of $\psi$, the vector $\boldsymbol{\eta}$ is

$$
\boldsymbol{\eta}=(\operatorname{sech} \psi \cos \phi, \operatorname{sech} \psi \sin \phi, \tanh \psi) .
$$

The natural appearance of the hyperbolic tangent in $\eta^{(z)}=\cos \theta$, already points to an inherent possibility of domain walls in the magnetic system, as will be discussed below.

\section{Analysis of equations (15) using complex variables}

Multi-instantons were originally derived [3] from equations (13), by defining the variable $M(x, t)=\cot (\theta / 2) \exp (\mathrm{i} \phi)=M_{1}(\theta, \phi)+\mathrm{i} M_{2}(\theta, \phi)$, whereupon it is possible to show that $M_{1 x}=M_{2 t}$ and $M_{1 t}=-M_{2 x}$. These are CRRs between two real functions $M_{1}$ and $M_{2}$, so that $M(\zeta)$ is analytic in the $\zeta=x+\mathrm{i} t$ complex plane. The simplest choice $M=\left(\zeta-\zeta_{o}\right)^{n}$ then yields the multi-instanton solution. Note that because $M_{1}$ and $M_{2}$ are mixed functions of the two angles $\theta$ and $\phi$, the solutions for $\cos \theta$ is in terms of both $M_{1}$ and $M_{2}$, making the analysis a little involved. The advantage of using the variable $\psi$ is that we obtain the CRRs (equations (15)) which separate the two variables in a natural way. Hence, any analytic fuction $\Phi \equiv \psi+\mathrm{i} \phi$ solves equations (15), and therefore the BPE. In the new variables, the $n$-instanton solution would correspond to $\Phi(\zeta)=n \ln \left(\zeta-\zeta_{0}\right)$. However, for deriving travelling twist solutions, this type of analysis is not very convenient. For example, for the analytic function $\Phi(\zeta)=\left(\zeta-\zeta_{0}\right) / \Gamma$

$$
\cos \theta=\eta^{(z)}=\tanh \left[\left(x-x_{0}\right) / \Gamma\right] \quad \phi=\left(t-t_{0}\right) / \Gamma .
$$

Inspection of this solution shows that at any given instant of time $t$, the vector $\boldsymbol{\eta}$ which lies along the positive $z$ direction at $x \rightarrow-\infty$ gradually twists down, acquiring $x$ and $y$ components until, at a certain intermediate fixed $x=x_{0}$, it lies fully in the $(x, y)$ plane. From then on it continues twisting down until it finally comes to rest along the negative $z$ direction as $x \rightarrow+\infty$. Further, the angle $\phi=\left(t-t_{0}\right) / \Gamma$ also twists, rotating about the $z$ axis with an angular velocity $(\Gamma)^{-1}$. The sign of $\eta^{(z)}$ changes just once as $x$ goes from $-\infty$ to $+\infty$ in such a way that the position $x_{0}$ at which it changes sign (i.e. the location of the domain wall) is independent of time. This is therefore defined to be a single non-travelling twist or nontravelling domain wall. (Hereafter, we will use the terms 'twist' and 'domain wall' interchangeably.)

We can now generalize this form to any nontravelling $N$-twist (multi-twist) solution:

$$
\Phi(\zeta)=\prod_{j=1}^{N}\left(\zeta-\zeta_{j}\right) / \Gamma_{j}
$$

Here, $\eta^{(z)}$ changes sign $N$ times, as $x$ goes from $-\infty$ to $+\infty$ in such a way that the $x$ values at which sign changes occur are all independent of time. To obtain travelling twists, we analyse equations (15) without going over to the complex $\zeta$ plane. In the next section we derive the travelling single twist and bi-twist solutions. 


\section{Travelling single-twist and bi-twist solutions}

It is convenient to use moving coordinates to solve equations (15) for travelling twists:

$$
\xi \equiv c \sqrt{1+\omega^{2}}(x-v t) \quad \sigma \equiv c \sqrt{1+v^{2}}(\omega x+t) .
$$

Here, $v$ and $-1 / \omega$ are independent velocities and $c$ is a global rescaling factor. In terms of $\xi$ and $\sigma$, relations (15) become

$$
\begin{array}{lc}
\psi_{\xi}=\alpha \phi_{\xi}-\beta \phi_{\sigma} & \psi_{\sigma}=\beta \phi_{\xi}-\alpha \phi_{\sigma} \\
\alpha \equiv(\omega-v) / \mu & \beta \equiv \gamma / \mu
\end{array}
$$

where $\gamma \equiv \sqrt{\left(1+v^{2}\right) /\left(1+\omega^{2}\right)}$ and $\mu \equiv 1+v \omega$. Note that $c$ scales out, which means that relations (19) are invariant to a uniform scaling of the entire plane. Eliminating either $\psi$ or $\phi$ we have

$$
\mathcal{L}\{\psi\}=\mathcal{L}\{\phi\}=0
$$

where the operator $\mathcal{L}$ is

$$
\mathcal{L} \equiv \partial_{\xi \xi}-2(\alpha / \beta) \partial_{\xi \sigma}+\partial_{\sigma \sigma} .
$$

We now seek particular solutions of the form

$$
\begin{aligned}
& \psi(\xi, \sigma)=a_{1} \xi^{2}+a_{2} \xi \sigma+a_{3} \sigma^{2}+a_{4} \xi+a_{5} \sigma+\psi_{0} \\
& \phi(\xi, \sigma)=b_{1} \xi^{2}+b_{2} \xi \sigma+b_{3} \sigma^{2}+b_{4} \xi+b_{5} \sigma+\phi_{0} .
\end{aligned}
$$

On using (15), we find that the twelve coefficients appearing in (22) must satisfy six independent relations and therefore only six of them are independent. The form (22) is an unusual solution for $\cos \theta=\tanh \psi$. Although hyperbolic functions abound in nonlinear problems, their arguments are generically linear, rather than quadratic, in space and time coordinates. Moreover, the freedom in the choice of the coefficients, $a_{j}$, leaves room for many interesting combinations, some of which are explored next.

\subsection{A travelling single twist}

Choosing real $v$ and $\omega$ and setting $a_{1}=a_{2}=a_{3}=0$ in equation (22) we obtain $\cos \theta=\tanh \left(a_{4} \xi+a_{5} \sigma+\psi_{0}\right)$ and $\phi-\phi_{0}=-\left[\left(\alpha a_{4}-\beta a_{5}\right) \xi+\left(\beta a_{4}-\alpha a_{5}\right) \sigma\right]$. For $x \rightarrow \pm \infty$, $\cos \theta \rightarrow \pm 1(\theta \rightarrow \pm \pi)$ and therefore this is a twist solution in $\theta$. While $\theta$ changes abruptly across the location of the node of $\psi$, the angle $\phi$ rotates linearly with $x$ and $t$. The propagation speeds of $\psi$ and $\phi$ are

$$
V_{\psi}=[v-b \gamma] /[1+b \omega \gamma] \quad V_{\phi}=[b v+\gamma] /[b-\omega \gamma]
$$

where $b=a_{5} / a_{4}$. Analysis of these relations shows that $\psi$ and $\phi$ cannot propagate at the same speed, implying that $\phi$ rotates constantly across the domain wall. We note that the special case $\omega=v$ and $a_{5}=0$ yields $V_{\psi}=v$ and $V_{\phi}=-1 / v$ and reproduces the single travelling twist solution [4] mentioned in the introduction. That particular single twist has the form $\cos \theta=-\tanh (k x-\omega t)$ and $\phi=(\omega x+k t)$ and it follows that

$\boldsymbol{\eta}=[\operatorname{sech}(k x-\omega t) \cos (\omega x+k t), \operatorname{sech}(k x-\omega t) \sin (\omega x+k t),-\tanh (k x-\omega t)]$.

In contrast to the nontravelling twist of the last section, this is a travelling single-twist solution for $\boldsymbol{\eta}$ whose $x$-position travels at a velocity $V_{\psi}$, while the $\phi$-field travels at a velocity $V_{\phi}$. In other words, a travelling single twist is a moving domain wall with a 'spin-wave' (but with a varying amplitude) mode present within the wall thickness. 


\subsection{Travelling bi-twist solutions}

We now proceed to discuss more complex solutions. Rewriting $\psi$ and $\phi$ in the quadratic form:

$$
\left(\begin{array}{l}
\psi \\
\phi
\end{array}\right)=\left(\begin{array}{l}
A \\
D
\end{array}\right) x^{2}+\left(\begin{array}{l}
B(t) \\
E(t)
\end{array}\right) x+\left(\begin{array}{l}
C(t) \\
F(t)
\end{array}\right)
$$

and applying equation (15), we find that $A$ and $D$ are arbitrary constants, $B=-2 D t+B_{0}$, $E=2 A t+E_{0}, C=-A t^{2}-E_{0} t+C_{0}$, and $F=-D t^{2}+B_{0} t+F_{0}$. Rearranging these relations we get

$$
\left(\begin{array}{l}
\psi \\
\phi
\end{array}\right)=\left(\begin{array}{l}
A \\
D
\end{array}\right)\left[x+\left(\begin{array}{l}
\alpha(t) \\
\gamma(t)
\end{array}\right)\right]\left[x+\left(\begin{array}{l}
\beta(t) \\
\delta(t)
\end{array}\right)\right]
$$

where $\alpha$ and $\beta$ ( $\gamma$ and $\delta$ ) are the (time-dependent) roots of the quadratic forms of $\psi(\phi)$. When the roots are real the solution describes a pulse in $\cos \theta$, whose width is

$$
W_{\psi}=2 \Delta(t) / A
$$

where $\Delta^{2}(t)=\left(A^{2}+D^{2}\right) t^{2}+\left(E_{0} A-B_{0} D\right) t+\left(B_{0}^{2} / 4-C_{0} A\right)$. Since $A$ and $D$ are real then after some time $\Delta$ always increases with time and therefore even roots that are initially complex eventually become real. This also implies a kind of instability of pulses in the sense that an initial pulse always broadens with time. This broadening happens at an asymptotic rate of

$$
V_{W}=2 \sqrt{1+D^{2} / A^{2}}
$$

For example, suppose that we start the system at $t_{0}=0$. Then from equation (25) we have

$$
\cos \theta=\tanh \left(A x^{2}+B_{0} x+C_{0}\right)
$$

and

$$
\phi=D x^{2}+E_{0} x+F_{0} .
$$

For $A>0(A<0)$ we have $\eta^{(z)} \rightarrow 1(-1)$ as $|x| \rightarrow \infty$ and the evolution is uniquely determined by equation (26). For $A>0$, the minimum of $\psi$ is at $x_{0}=\left(B_{0} / 2-D t\right) / A$ and the value of $\eta^{(z)}$ at $x_{0}$ decreases with time to rapidly approach -1 :

$$
\eta_{\min }^{(z)}=\tanh \left[-A \Delta^{2}(t)\right]
$$

Thus even a very small initial quadratic corrugation in $\eta^{(z)}$ eventually evolves to -1 and there is an infinite broadening of the domain of $\eta^{(z)}=-1$ within a uniform state of $\eta^{(z)}=1$, with the domain centre travelling at a speed of $-D / A$. For $A<0$ a mirror-image situation occurs where the $\eta^{(z)}=1$ state grows inside the $\eta^{(z)}=-1$ state. An important question is whether the domain walls are stable and remain sharp since a domain wall that also broadens with time loses its meaning as such. Defining then the thickness of the domain wall, $w$, as the range of $x$ over which $\eta^{(z)}$ changes from -0.8 to 0.8 , we find that the walls sharpen with time, $w=8 \ln 3 \sqrt{D^{2}+A^{2}} / t \rightarrow 0$. This is shown in figure 1 , for $A=D=1 / \sqrt{2}, C_{0}=F_{0}=0$, $B_{0}=2$ and $E_{0}=1$. To the best of our knowledge this is the first time that nonlinear solutions of this kind have been derived for this problem.

\section{Generalization to travelling multi-twist solutions}

In this section, we show that the single-twist and bi-twist solutions discussed in section 4 are but the first two members of an infinite hierarchy of solutions. To this end, we write $\psi$ and $\phi$ in the form

$$
\left(\begin{array}{l}
\psi \\
\phi
\end{array}\right)=\sum_{k=0}^{N}\left(\begin{array}{c}
P_{N-k} \\
Q_{N-k}
\end{array}\right) x^{k}
$$




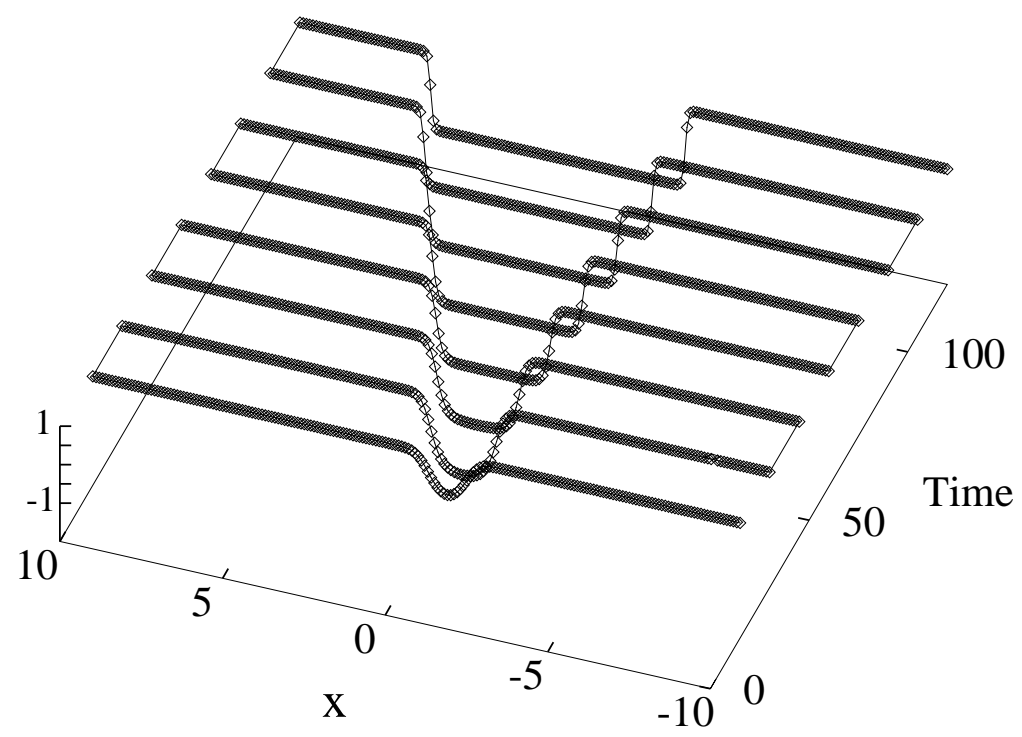

Figure 1. A bi-twist analytic solution for $\eta^{(z)}=\cos \theta$.

where $P_{n}$ and $Q_{n}$ are polynomials of degree $n$ in the time variable $t$. Using equation (15), each of these polynomials has to satisfy the following second-order ODE:

$$
\ddot{R}_{k+2, t t}+(N-k-1)(N-k) R_{k}=0
$$

where $R$ is either $P(t)$ or $Q(t)$. We use these equations to construct the polynomials recursively. It can be readily shown that, other than their constant terms, the $Q$ polynomials are completely determined by the $P$ polynomials. Since each second-order ODE introduces two constants, the solution has $2 N+2$ arbitrary constants, $P_{0}, P_{1}(0), \ldots, P_{N}(0), Q_{0}, Q_{1}(0), \ldots, Q_{N}(0)$, determined by the initial data. The solutions for $\eta^{(z)}=\cos \theta$,

$$
\eta^{(z)}=\tanh \left(\sum_{k=0}^{N} P_{N-k} x^{k}\right)
$$

describe many domain walls travelling along the chain. A wall occurs wherever $\psi$ has a real root in the variable $x$ and hence the number of walls at any time is, at most, $N$. Figure 2 shows a typical such solution for $N=3$, where an initially slightly distorted single twist develops into three walls. In the general case it is the highest order term in $t$ that dominates asymptotically and $\psi(t \rightarrow \infty)$ converges to the form

$$
\psi=t^{N} \sum_{k=0}^{N} c_{N-k}(x / t)^{k}=P_{0} \prod_{i=1}^{N}\left(x-v_{i} t\right)
$$

where $c_{n}$ is the (real) coefficient of $t^{n}$ in $P_{n}(t)$. Note that, in principle, the roots $v_{i}$ may be complex, in which case there is no actual wall. When all the $v_{i}$ are real, the solution has exactly $N$ walls moving at velocities $v_{i}$ that are interdependent. For example, for $N=2$ we have seen that the velocities are related by $v_{1}=-1 / v_{2}$. In (32) the distance between any two neighbouring walls increases with time, which represents continuously growing domains of alternating polar orientations. This may be regarded as repulsion forces between twists and anti-twists in this system. 


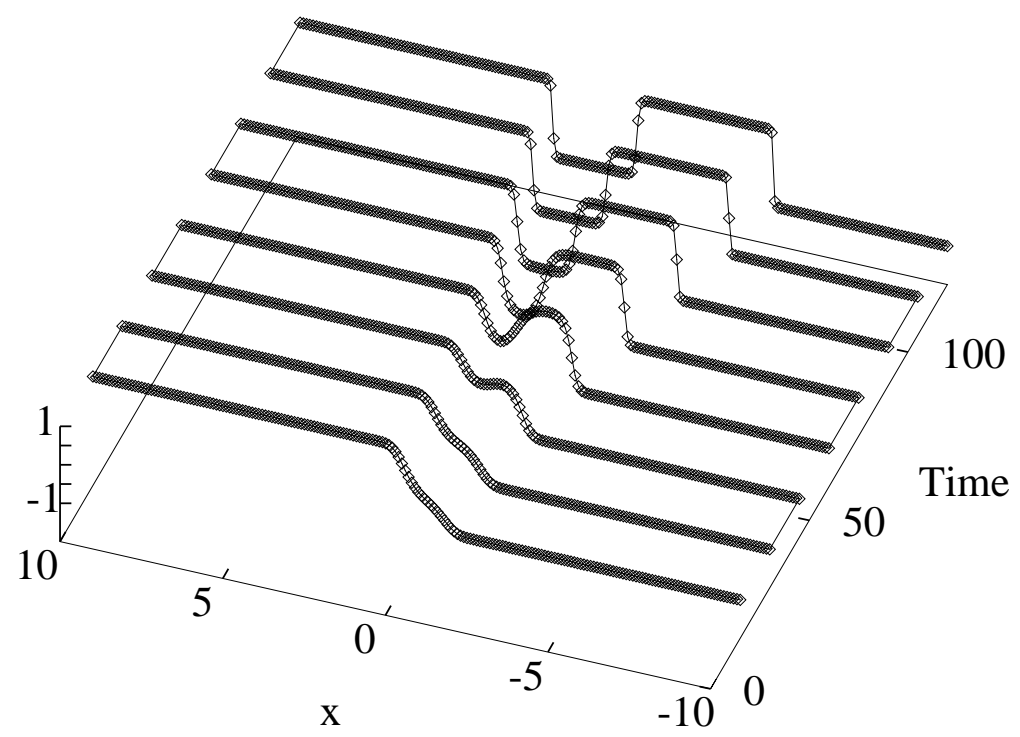

Figure 2. A three-twist solution for $\eta^{(z)}$ : Note the production of three walls from one initial wall.

Since the coefficients of the polynomials are time dependent, at intermediate times twists may be created and annihilated. However, they must do so in pairs. Creation occurs when a local minimum of $\psi(x)$ downshifts with time until it intersects with the $x=0$ axis whereupon it generates two real roots that initiate two domain walls. Further downshift moves the domain walls apart. Annihilation is the opposite process when a local minimum of $\psi$ moves upwards relative to the real axis until the real roots meet and disappear. If the case $N=2$ is generic then creation is more likely than anihilation in the long run and walls will be generated until their number reaches $N$.

\section{Summary and conclusions}

In this paper, we have found exact $N$-twist solutions for the BPE, which describes (a) the evolution dynamics of the spin vectors in an Heisenberg AFM spin chain at low energies (equation (11)) and (b) the stationary solutions for the classical continuous two-dimensional Heisenberg FM. This equation also describes a wide class of space curves moving in three dimensions [2]. The solutions found here are markedly different from the known multiinstantons [3] and merons [8]. Using the bi-twist solution given in section 4, we have plotted the corresponding moving curve in figure 3 . (The moving curve corresponding to the singletwist solution can be found in [2].)

Our results are significant in view of the fast technological advances made in recent years in the fabrication of a wide variety of low-dimensional magnets. In particular, these have inspired much interest in the nonlinear excitations that occur in Heisenberg spin chains [7] to which our solutions directly apply. In two-dimensional FM layers the multi-twists correspond to static spin textures, while in the AFM chain they describe moving domain walls. We remark that the analysis presented here also applies to certain inhomogeneous AFM chains, as discussed in [2]. Specifically, in that case these solutions are given in terms of transformed variables that are determined by the particular inhomogeneity. 


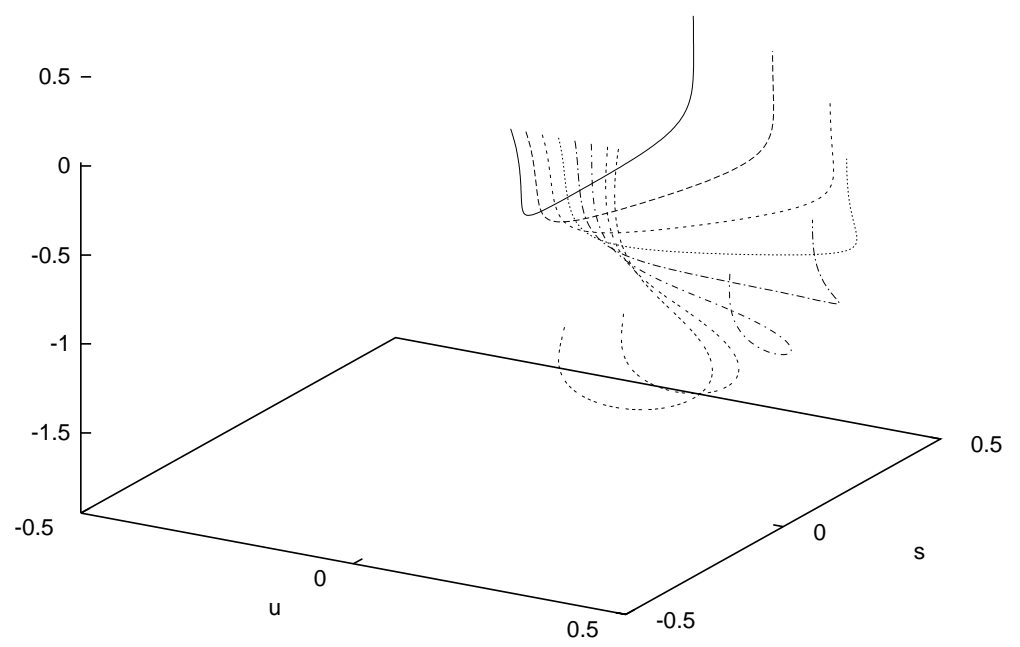

Figure 3. A bi-twist moving curve solution of the BPE (equation (4)).

Our solutions are relevant in yet another context. By identifying the vector $\boldsymbol{\eta}$ with the tangent to a moving curve and using Lamb's formalism [1], equation (11) is gauge-equivalent to the following nonlinear integro-differential equation [4]:

$$
\mathrm{i} \Psi_{t}+\Psi_{x}-\Psi \int_{-\infty}^{x}|\Psi|^{2} \mathrm{~d} x^{\prime}=0
$$

where $\Psi=(K / 2) \exp \left\{\mathrm{i} \int^{x}\left(\mathrm{~d} x^{\prime} / K^{2}\right) \boldsymbol{\eta} \cdot\left(\boldsymbol{\eta}_{x^{\prime}} \times \boldsymbol{\eta}_{x^{\prime} x^{\prime}}\right)\right\}$ is a complex function and $K^{2}=4|\Psi|^{2}=$ $\left|\boldsymbol{\eta}_{x}\right|^{2}$. Thus, in that context, the multi-twist solutions for $\boldsymbol{\eta}$ carry over into a hierarchy of solutions for $\Psi(x, t)$.

The boundary conditions determine whether the number of twists is even $\left(\eta^{(z)}( \pm \infty)=1\right)$ or odd $\left(\eta^{(z)}( \pm \infty)= \pm 1\right)$. The asymptotic form (32), combined with equation (31), describes independent twists that propagate without change of shape and are thus reminiscent of kinksolitons. This similarity begs the intriguing question whether these solutions occur in other nonlinear systems. Finally, we comment that there are still questions of stability to be cleared in the following sense: while these solutions are certainly stable for the static two-dimensional FM case, it is not clear whether this is so for the AFM application. We hope to present a detailed analysis of this issue in a future report, as we believe that it is of much physical interest.

\section{Acknowledgments}

We thank A R Bishop, Philip Rosenau and V G Makhankov for helpful discussions.

\section{References}

[1] Lamb G L 1977 J. Math. Phys. 181654

[2] Radha Balakrishnan and Blumenfeld R 1997 J. Math. Phys. 385878

[3] Belavin A and Polyakov A M 1975 Pis. Zh. Eksp. Teor. Fiz. 22503 Belavin A and Polyakov A M 1975 JETP Lett. 22245

[4] Radha Balakrishnan, Bishop A R and Dandoloff R 1993 Phys. Rev. B 473108 Radha Balakrishnan, Bishop A R and Dandoloff R 1990 Phys. Rev. Lett. 642107 
[5] Nakamura K and Sasada T 1974 Phys. Lett. A 48321

Tjon J and Wright J 1977 Phys. Rev. B 153470

[6] Takhtajan L A 1977 Phys. Lett. A 64235

[7] Mikeska H J and Steiner M 1991 Adv. Phys. 40191

[8] Callan C G Jr, Dashen R F and Gross D J 1977 Phys. Lett. B 66375

Gross D J 1978 Nucl. Phys. B 132439 